\title{
Unbalanced Oil and Vinegar Signature Schemes
}

\author{
Aviad Kipnis ${ }^{1}$, Jacques Patarin ${ }^{2}$, and Louis Goubin ${ }^{2}$ \\ 1 NDS Technologies, 5 Hamarpe St. Har Hotzvim, Jerusalem - Israel, \\ akipnis@ndsisrael.com \\ 2 Bull SmartCards and Terminals, 68 route de Versailles - BP45, \\ 78431 Louveciennes Cedex - France, \\ $\{\mathrm{J}$. Patarin, L.Goubin\}@frlv.bull.fr
}

\begin{abstract}
In 16, J. Patarin designed a new scheme, called "Oil and Vinegar", for computing asymmetric signatures. It is very simple, can be computed very fast (both in secret and public key) and requires very little RAM in smartcard implementations. The idea consists in hiding quadratic equations in $n$ unknowns called "oil" and $v=n$ unknowns called "vinegar" over a finite field $K$, with linear secret functions. This original scheme was broken in [10 by A. Kipnis and A. Shamir. In this paper, we study some very simple variations of the original scheme where $v>n$ (instead of $v=n)$. These schemes are called "Unbalanced Oil and Vinegar" (UOV), since we have more "vinegar" unknowns than "oil" unknowns. We show that, when $v \simeq n$, the attack of [10] can be extended, but when $v \geq 2 n$ for example, the security of the scheme is still an open problem. Moreover, when $v \simeq \frac{n^{2}}{2}$, the security of the scheme is exactly equivalent (if we accept a very natural but not proved property) to the problem of solving a random set of $n$ quadratic equations in $\frac{n^{2}}{2}$ unknowns (with no trapdoor). However, we show that (in characteristic 2) when $v \geq n^{2}$, finding a solution is generally easy. Then we will see that it is very easy to combine the Oil and Vinegar idea and the HFE schemes of 14. The resulting scheme, called HFEV, looks at the present also very interesting both from a practical and theoretical point of view. The length of a UOV signature can be as short as 192 bits and for HFEV it can be as short as 80 bits.
\end{abstract}

Note: An extended version of this paper can be obtained from the authors.

\section{Introduction}

Since 1985, various authors (see [7], [9], 12], [14], [16], [17], 18], 21] for example) have suggested some public key schemes where the public key is given as a set of multivariate quadratic (or higher degree) equations over a small finite field $K$.

The general problem of solving such a set of equations is NP-hard (cf [8]) (even in the quadratic case). Moreover, when the number of unknowns is, say, $n \geq$ 16 , the best known algorithms are often not significantly better than exhaustive search (when $n$ is very small, Gröbner bases algorithms are more efficient, cf [6]). 
The schemes are often very efficient in terms of speed or RAM required in a smartcard implementation. (However, the length of the public key is generally $\geq 1$ Kbyte. Nevertheless, it is sometimes useful to notice that secret key computations can be performed without the public key). The most serious problem is that, in order to introduce a trapdoor (to allow the computation of signatures or to allow the decryption of messages when a secret is known), the generated set of public equations generally becomes a small subset of all the possible equations and, in many cases, the algorithms have been broken. For example [7] was broken by their authors, and [12, 16, 21 were broken. However, many schemes are still not broken (for example [14], 17], [18], 20]), and also in many cases, some very simple variations have been suggested in order to repair the schemes. Therefore, at the present, we do not know whether this idea of designing public key algorithms with multivariate polynomials over small finite fields is a very powerful idea (where only some too simple schemes are insecure) or not.

In this paper, we will present two new schemes: UOV and HFEV. UOV is a very simple scheme: the original Oil and Vinegar signature scheme (of [16]) was broken (see 10]), but if we have significantly more "vinegar" unknowns than "oil" unknowns (a definition of the "oil" and "vinegar" unknowns can be found in section 2), then the attack of [10] does not work and the security of this more general scheme (called UOV) is still an open problem. We will also study Oil and Vinegar schemes of degree three (instead of two). Then, we will present another scheme, called HFEV. HFEV combines the ideas of HFE (of [14) and of vinegar variables. HFEV looks more efficient than the original HFE scheme. Finally, in section 13, we present what we know about the main schemes in this area of multivariate polynomials.

\section{The (Original and Unbalanced) Oil and Vinegar of Degree Two}

Let $K=\mathbf{F}_{q}$ be a small finite field (for example $K=\mathbf{F}_{2}$ ). Let $n$ and $v$ be two integers. The message to be signed (or its hash) is represented as an element of $K^{n}$, denoted by $y=\left(y_{1}, \ldots, y_{n}\right)$. Typically, $q^{n} \simeq 2^{128}$ (in section 8 , we will see that $q^{n} \simeq 2^{64}$ is also possible). The signature $x$ is represented as an element of $K^{n+v}$ denoted by $x=\left(x_{1}, \ldots, x_{n+v}\right)$.

\section{Secret Key}

The secret key is made of two parts:

1. A bijective and affine function $s: K^{n+v} \rightarrow K^{n+v}$. By "affine", we mean that each component of the output can be written as a polynomial of degree one in the $n+v$ input unknowns, and with coefficients in $K$.

2. A set $(\mathcal{S})$ of $n$ equations of the following type:

$$
\forall i, 1 \leq i \leq n, y_{i}=\sum \gamma_{i j k} a_{j} a_{k}^{\prime}+\sum \lambda_{i j k} a_{j}^{\prime} a_{k}^{\prime}+\sum \xi_{i j} a_{j}+\sum \xi_{i j}^{\prime} a_{j}^{\prime}+\delta_{i}(\mathcal{S})
$$


The coefficients $\gamma_{i j k}, \lambda_{i j k}, \xi_{i j}, \xi_{i j}^{\prime}$ and $\delta_{i}$ are the secret coefficients of these $n$ equations. The values $a_{1}, \ldots, a_{n}$ (the "oil" unknowns) and $a_{1}^{\prime}, \ldots, a_{v}^{\prime}$ (the "vinegar" unknowns) lie in $K$. Note that these equations $(\mathcal{S})$ contain no terms in $a_{i} a_{j}$.

\section{Public Key}

Let $A$ be the element of $K^{n+v}$ defined by $A=\left(a_{1}, \ldots, a_{n}, a_{1}^{\prime}, \ldots, a_{v}^{\prime}\right) . A$ is transformed into $x=s^{-1}(A)$, where $s$ is the secret, bijective and affine function from $K^{n+v}$ to $K^{n+v}$. Each value $y_{i}, 1 \leq i \leq n$, can be written as a polynomial $P_{i}$ of total degree two in the $x_{j}$ unknowns, $1 \leq j \leq n+v$. We denote by $(\mathcal{P})$ the set of the following $n$ equations:

$$
\forall i, 1 \leq i \leq n, y_{i}=P_{i}\left(x_{1}, \ldots, x_{n+v}\right)
$$

These $n$ quadratic equations $(\mathcal{P})$ (in the $n+v$ unknowns $x_{j}$ ) are the public key.

\section{Computation of a Signature (with the Secret Key)}

The computation of a signature $x$ of $y$ is performed as follows:

Step 1: We find $n$ unknowns $a_{1}, \ldots, a_{n}$ of $K$ and $v$ unknowns $a_{1}^{\prime}, \ldots, a_{v}^{\prime}$ of $K$ such $\overline{\text { that the }} n$ equations $(\mathcal{S})$ are satisfied. This can be done as follows: we randomly choose the $v$ vinegar unknowns $a_{i}^{\prime}$, and then we compute the $a_{i}$ unknowns from $(\mathcal{S})$ by Gaussian reductions (because - since there are no $a_{i} a_{j}$ terms - the $(\mathcal{S})$ equations are affine in the $a_{i}$ unknowns when the $a_{i}^{\prime}$ are fixed).

Remark: If we find no solution, then we simply try again with new random vinegar unknowns. After very few tries, the probability of obtaining at least one solution is very high, because the probability for a $n \times n$ matrix over $\mathbf{F}_{q}$ to be invertible is not negligible. (It is exactly $\left(1-\frac{1}{q}\right)\left(1-\frac{1}{q^{2}}\right) \ldots\left(1-\frac{1}{q^{n-1}}\right)$. For $q=2$, this gives approximately $30 \%$, and for $q>2$, this probability is even larger.)

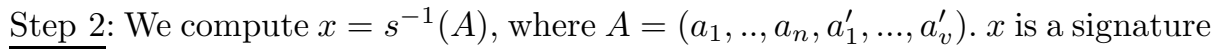
of $y$.

\section{Public Verification of a Signature}

A signature $x$ of $y$ is valid if and only if all the $(\mathcal{P})$ are satisfied. As a result, no secret is needed to check whether a signature is valid: this is an asymmetric signature scheme.

Note: The name "Oil and Vinegar" comes from the fact that - in the equations $(\mathcal{S})$ - the "oil unknowns" $a_{i}$ and the "vinegar unknowns" $a_{j}^{\prime}$ are not all mixed together: there are no $a_{i} a_{j}$ products. However, in $(\mathcal{P})$, this property is hidden by the "mixing" of the unknowns by the $s$ transformation. Is this property "hidden enough" ? In fact, this question exactly means: "is the scheme secure ?". When 
$v=n$, we call the scheme "Original Oil and Vinegar", since this case was first presented in [16]. This case was broken in [10]. It is very easy to see that the cryptanalysis of [10] also works, exactly in the same way, when $v<n$. However, the cases $v>n$ are, as we will see, much more difficult. When $v>n$, we call the scheme "Unbalanced Oil and Vinegar".

\section{Cryptanalysis of the Case $v=n$ (from [10])}

The idea of the attack of [10] is essentially the following: In order to separate the oil variables and the vinegar variables, we look at the quadratic forms of the $n$ public equations of $(\mathcal{P})$, we omit for a while the linear terms. Let $G_{i}$ for $1 \leq i \leq n$ be the respective matrix of the quadratic form of $P_{i}$ of the public equations $(\mathcal{P})$. The quadratic part of the equations in the set $(\mathcal{S})$ is represented as a quadratic form with a corresponding $2 n \times 2 n$ matrix of the form : $\left(\begin{array}{ll}0 & A \\ B & C\end{array}\right)$, the upper left $n \times n$ zero submatrix is due to the fact that an oil variable is not multiplied by an oil variable. After hiding the internal variables with the linear function $s$, we get a representation for the matrices $G_{i}=S\left(\begin{array}{ll}0 & A_{i} \\ B_{i} & C_{i}\end{array}\right) S^{t}$, where $S$ is an invertible $2 n \times 2 n$ matrix.

Definition 3.1: We define the oil subspace to be the linear subspace of all vectors in $K^{2 n}$ whose second half contains only zeros.

Definition 3.2: We define the vinegar subspace as the linear subspace of all vectors in $K^{2 n}$ whose first half contains only zeros.

Lemma 1. Let $E$ and $F$ be a $2 n \times 2 n$ matrices with an upper left zero $n \times n$ submatrix. If $F$ is invertible then the oil subspace is an invariant subspace of $E F^{-1}$.

Proof: see [10].

Definition 3.4: For an invertible matrix $G_{j}$, define $G_{i j}=G_{i} G_{j}^{-1}$.

Definition 3.5: Let $O$ be the image of the oil subspace by $S^{-1}$.

In order to find the oil subspace, we use the following theorem:

Theorem 3.1. $O$ is a common invariant subspace of all the matrices $G_{i j}$.

\section{Proof:}

$$
G_{i j}=S\left(\begin{array}{ll}
0 & A_{i} \\
B_{i} & C_{i}
\end{array}\right) S^{t}\left(S^{t}\right)^{-1}\left(\begin{array}{cc}
0 & A_{j} \\
B_{j} & C_{j}
\end{array}\right)^{-1} S^{-1}=S\left(\begin{array}{ll}
0 & A_{i} \\
B_{i} & C_{i}
\end{array}\right)\left(\begin{array}{ll}
0 & A_{j} \\
B_{j} & C_{j}
\end{array}\right)^{-1} S^{-1}
$$

The two inner matrices have the form of $E$ and $F$ in lemma 1. Therefore, the oil subspace is an invariant subspace of the inner term and $O$ is an invariant 
subspace of $G_{i} G_{j}^{-1}$. The problem of finding common invariant subspace of set of matrices is studied in [10]. Applying the algorithms in [10] gives us $O$. We then pick $V$ to be an arbitrary subspace of dimension $n$ such that $V+O=K^{2 n}$, and they give an equivalent oil and vinegar separation. Once we have such a separation, we bring back the linear terms that were omitted, we pick random values for the vinegar variables and left with a set of $n$ linear equations with $n$ oil variables.

Note: Lemma 1 is not true any more when $v>n$. The oil subspace is still mapped by $E$ and $F$ into the vinegar subspace. However $F^{-1}$ does not necessary maps the image by $E$ of the oil subspace back into the oil subspace and this is why the cryptanalysis of the original oil and vinegar is not valid for the unbalanced case.

\section{Cryptanalysis when $v>n$ and $v \simeq n$}

In this section, we will describe a modification of the above attack, that is applicable as long as $v-n$ is small (more precisely the expected complexity of the attack is approximately $\left.q^{(v-n)-1} \cdot n^{4}\right)$.

Definition 4.1: We define in this section the oil subspace to be the linear subspace of all vectors in $K^{n+v}$ whose last $v$ coordinates are only zeros.

Definition 4.2: We define in this section the vinegar subspace to be the linear subspace of all vectors in $K^{n+v}$ whose first $n$ coordinates are only zeros.

Here in this section, we start with the homogeneous quadratic terms of the equations: we omit the linear terms for a while. The matrices $G_{i}$ have the representation

$$
G_{i}=S\left(\begin{array}{ll}
0 & A_{i} \\
B_{i} & C_{i}
\end{array}\right) S^{t}
$$

where the upper left matrix is the $n \times n$ zero matrix, $A_{i}$ is a $n \times v$ matrix, $B_{i}$ is a $v \times n$ matrix, $C_{i}$ is a $v \times v$ matrix and $S$ is a $(n+v) \times(n+v)$ invertible linear matrix.

Definition 4.3: Define $E_{i}$ to be $\left(\begin{array}{ll}0 & A_{i} \\ B_{i} & C_{i}\end{array}\right)$.

Lemma 2. For any matrix $E$ that has the form $\left(\begin{array}{ll}0 & A \\ B & C\end{array}\right)$, the following holds:

a) E transforms the oil subspace into the vinegar subspace.

b) If the matrix $E^{-1}$ exists, then the image of the vinegar subspace by $E^{-1}$ is a subspace of dimension $v$ which contains the $n$-dimensional oil subspace in $i t$. 
Proof: a) follows directly from the definition of the oil and vinegar subspaces. When a) is given then b) is immediate.

The algorithm we propose is probabilistic. It looks for an invariant subspace of the oil subspace after it is transformed by $S$. The probability for the algorithm to succeed on the first try is small. Therefore we need to repeat it with different inputs. We use the following property: any linear combination of the matrices $E_{1}, \ldots, E_{n}$ is also of the form $\left(\begin{array}{ll}0 & A \\ B & C\end{array}\right)$. The following theorem explains why an invariant subspace may exist with a certain probability.

Theorem 4.1. Let $F$ be an invertible linear combination of the matrices $E_{1}$, $\ldots, E_{n}$. Then for any $k$ such that $E_{k}^{-1}$ exists, the matrix $F E_{k}^{-1}$ has a non trivial invariant subspace which is also a subspace of the oil subspace, with probability not less than $\frac{q-1}{q^{2 d}-1}$ for $d=v-n$.

Proof: See the extended version of this paper.

Note: It is possible to get a better result for the expected number of eigenvectors and with much less effort: $I_{1}$ is a subspace with dimension not less than $n-d$ and is mapped by $F E_{k}^{-1}$ into a subspace with dimension $n$. The probability for a non zero vector to be mapped to a non zero multiple of itself is $\frac{q-1}{q^{n}-1}$. To get the expected value, we multiply it by the number of non zero vectors in $I_{1}$. It gives a value which is not less than $\frac{(q-1)\left(q^{n-d}-1\right)}{q^{n}-1}$. Since every eigenvector is counted $q-1$ times, then the expected number of invariant subspcaes of dimension 1 is not less than $\frac{q^{n-d}-1}{q^{n}-1} \sim q^{-d}$.

We define $O$ as in section 3 and we get the following result for $O$ :

Theorem 4.2. Let $F$ be an invertible linear combination of the matrices $G_{1}$, $\ldots, G_{n}$. Then for any $k$ such that $G_{k}^{-1}$ exists, the matrix $F G_{k}^{-1}$ has a non trivial invariant subspace, which is also a subspace of $O$ with probability not less than $\frac{q-1}{q^{2 d}-1}$ for $d=v-n$.

\section{Proof:}

$$
\begin{gathered}
F G_{k}^{-1}=\left(\alpha_{1} G_{1}+\ldots+\alpha_{n} G_{n}\right) G_{k}^{-1} \\
=S\left(\alpha_{1} E_{1}+\ldots+\alpha_{n} E_{n}\right) S^{t}\left(S^{t}\right)^{-1} E_{k}^{-1} S^{-1}=S\left(\alpha_{1} E_{1}+\ldots+\alpha_{n} E_{n}\right) E_{k}^{-1} S^{-1} .
\end{gathered}
$$

The inner term is an invariant subspace of the oil subspace with the required probability. Therefore, the same will hold for $F G_{k}^{-1}$, but instead of a subspace of the oil subspace, we get a subspace of $O$.

\section{How to find $O$ ?}

We take a random linear combination of $G_{1}, \ldots, G_{n}$ and multiply it by an inverse of one of the $G_{k}$ matrices. Then we calculate all the minimal invariant subspaces of this matrix (a minimal invariant subspace of a matrix $A$ contains no non trivial invariant subspaces of the matrix $A$ - these subspaces corresponds 
to irreducible factors of the characteristic polynomial of $A$ ). This can be done in probabilistic polynomial time using standard linear algebra techniques. This matrix may have an invariant subspace wich is a subspace of $O$.

The following lemma enables us to distinguish between subspaces that are contained in $O$ and random subspaces.

Lemma 3. If $H$ is a linear subspace and $H \subset O$, then for every $x, y$ in $H$ and every $i, G_{i}(x, y)=0$ (here we regard $G_{i}$ as a bilinear form).

Proof: There are $x^{\prime}$ and $y^{\prime}$ in the oil subspace such that $x^{\prime}=x S$ and $y^{\prime}=y S$.

$$
G_{i}(x, y)=x S\left(\begin{array}{cc}
0 & A_{i} \\
B_{i} & C_{i}
\end{array}\right) S^{t} y^{t}=x^{\prime}\left(\begin{array}{cc}
0 & A_{i} \\
B_{i} & C_{i}
\end{array}\right)\left(y^{\prime}\right)^{t}=0 .
$$

The last term is zero because $x^{\prime}$ and $y^{\prime}$ are in the oil subspace.

Lemma 3 gives a polynomial test to distinguish between subspaces of $O$ and random subspaces. If the matrix we used has no minimal subspace which is also a subspace of $O$, then we pick another linear combination of $G_{1}, \ldots, G_{n}$, multiply it by an inverse of one of the $G_{k}$ matrices and try again. After repeating this process approximately $q^{d-1}$ times, we find with good probability at least one zero vector of $O$. We continue the process until we get $n$ independent vectors of $O$. These vectors span $O$. The expected complexity of the process is proportional to $q^{d-1} \cdot n^{4}$. We use here the expected number of tries until we find a non trivial invariant subspace and the term $n^{4}$ covers the computational linear algebra operations we need to perform for evey try.

\section{The Cases $v \simeq \frac{n^{2}}{2}\left(\right.$ or $\left.v \geq \frac{n^{2}}{2}\right)$}

\section{Property}

Let $(\mathcal{A})$ be a random set of $n$ quadratic equations in $(n+v)$ variables $x_{1}, \ldots, x_{n+v}$. (By "random" we mean that the coefficients of these equations are uniformly and randomly chosen). When $v \simeq \frac{n^{2}}{2}$ (and more generally when $v \geq \frac{n^{2}}{2}$ ), there is probably - for most of such $(\mathcal{A})$ - a linear change of variables $\left(x_{1}, \ldots, x_{n+v}\right) \mapsto$ $\left(x_{1}^{\prime}, \ldots, x_{n+v}^{\prime}\right)$ such that the set $\left(\mathcal{A}^{\prime}\right)$ of $(\mathcal{A})$ equations written in $\left(x_{1}^{\prime}, \ldots, x_{n+v}^{\prime}\right)$ is an "Oil and Vinegar" system (i.e. there are no terms in $x_{i}^{\prime} \cdot x_{j}^{\prime}$ with $i \leq n$ and $j \leq n)$.

\section{An Argument to Justify the Property}

Let

$$
\left\{\begin{aligned}
x_{1} & =\alpha_{1,1} x_{1}^{\prime}+\alpha_{1,2} x_{2}^{\prime}+\cdots+\alpha_{1, n+v} x_{n+v}^{\prime} \\
& \vdots \\
x_{n+v} & =\alpha_{n+v, 1} x_{1}^{\prime}+\alpha_{n+v, 2} x_{2}^{\prime}+\cdots+\alpha_{n+v, n+v} x_{n+v}^{\prime}
\end{aligned}\right.
$$


By writing that the coefficient in all the $n$ equations of $(\mathcal{A})$ of all the $x_{i}^{\prime} \cdot x_{j}^{\prime}$ $(i \leq n$ and $j \leq n)$ is zero, we obtain a system of $n \cdot n \cdot \frac{n+1}{2}$ quadratic equations in the $(n+v) \cdot n$ variables $\alpha_{i, j}(1 \leq i \leq n+v, 1 \leq j \leq n)$. Therefore, when $v \geq$ approximately $\frac{n^{2}}{2}$, we may expect to have a solution for this system of equations for most of $(\mathcal{A})$.

\section{Remarks:}

1. This argument is very natural, but this is not a complete mathematical proof.

2. The system may have a solution, but finding the solution might be a difficult problem. This is why an Unbalanced Oil and Vinegar scheme might be secure (for well chosen parameters): there is always a linear change of variables that makes the problem easy to solve, but finding such a change of variables might be difficult.

3. In section 7 , we will see that, despite the result of this section, it is not recommended to choose $v \geq n^{2}$ (at least in characteristic 2).

\section{Solving a Set of $n$ Quadratic Equations in $k$ Unknowns, $k>n$, Is NP-hard}

(See the extended version of this paper.)

\section{A Generally (but Not Always) Efficient Algorithm for Solving a Random Set of $n$ Quadratic Equations in $n^{2}$ (or More) Unknowns}

In this section, we describe an algorithm that solves a system of $n$ randomly chosen quadratic equations in $n+v$ variables, when $v \geq n^{2}$.

Let $(\mathcal{S})$ be the following system:

$$
\left\{\begin{aligned}
\sum_{1 \leq i \leq j \leq n+v} a_{i j 1} x_{i} x_{j}+\sum_{1 \leq i \leq n+v} b_{i 1} x_{i}+\delta_{1} & =0 \\
\sum_{1 \leq i \leq j \leq n+v} a_{i j n} x_{i} x_{j}+\sum_{1 \leq i \leq n+v} b_{i n} x_{i}+\delta_{n} & =0
\end{aligned}\right.
$$

The main idea of the algorithm consists in using a change of variables such as:

$$
\left\{\begin{aligned}
x_{1} & =\alpha_{1,1} y_{1}+\alpha_{2,1} y_{2}+\ldots+\alpha_{n+v, 1} y_{n+v} \\
& \vdots \\
x_{n+v} & =\alpha_{1, n+v} y_{1}+\alpha_{2, n+v} y_{2}+\ldots+\alpha_{n+v, n+v} y_{n+v}
\end{aligned}\right.
$$

whose $\alpha_{i, j}$ coefficients (for $1 \leq i \leq n, 1 \leq j \leq n+v$ ) are found step by step, in order that the resulting system $\left(\mathcal{S}^{\prime}\right)$ (written with respect to these new variables $\left.y_{1}, \ldots, y_{n+v}\right)$ is easy to solve. 
- We begin by choosing randomly $\alpha_{1,1}, \ldots, \alpha_{1, n+v}$.

- We then compute $\alpha_{2,1}, \ldots, \alpha_{2, n+v}$ such that $\left(\mathcal{S}^{\prime}\right)$ contains no $y_{1} y_{2}$ terms. This condition leads to a system of $n$ linear equations on the $(n+v)$ unknowns $\alpha_{2, j}(1 \leq j \leq n+v)$ :

$$
\sum_{1 \leq i \leq j \leq n+v} a_{i j k} \alpha_{1, i} \alpha_{2, j}=0 \quad(1 \leq k \leq n) .
$$

- We then compute $\alpha_{3,1}, \ldots, \alpha_{3, n+v}$ such that $\left(\mathcal{S}^{\prime}\right)$ contains neither $y_{1} y_{3}$ terms, nor $y_{2} y_{3}$ terms. This condition is equivalent to the following system of $2 n$ linear equations on the $(n+v)$ unknowns $\alpha_{3, j}(1 \leq j \leq n+v)$ :

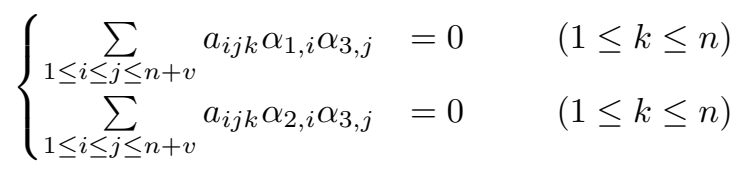

- ...

- Finally, we compute $\alpha_{n, 1}, \ldots, \alpha_{n, n+v}$ such that $\left(\mathcal{S}^{\prime}\right)$ contains neither $y_{1} y_{n}$ terms, nor $y_{2} y_{n}$ terms, ..., nor $y_{n-1} y_{n}$ terms. This condition gives the following system of $(n-1) n$ linear equations on the $(n+v)$ unknowns $\alpha_{n, j}$ $(1 \leq j \leq n+v)$ :

$$
\left\{\begin{array}{rlr}
\sum_{1 \leq i \leq j \leq n+v} a_{i j k} \alpha_{1, i} \alpha_{n, j} & =0 & (1 \leq k \leq n) \\
\sum_{1 \leq i \leq j \leq n+v} a_{i j k} \alpha_{n-1, i} \alpha_{n, j} & =0 & (1 \leq k \leq n)
\end{array}\right.
$$

In general, all these linear equations provide at least one solution (found by Gaussian reductions). In particular, the last system of $n(n-1)$ equations and $(n+v)$ unknowns generally gives a solution, as soon as $n+v>n(n-1)$, i.e. $v>n(n-2)$, which is true by hypothesis.

Moreover, the $n$ vectors $\left(\begin{array}{c}\alpha_{1,1} \\ \vdots \\ \alpha_{1, n+v}\end{array}\right), \ldots,\left(\begin{array}{c}\alpha_{n, 1} \\ \vdots \\ \alpha_{n, n+v}\end{array}\right)$ are very likely to be linearly independent for a random quadratic system $(\mathcal{S})$.

The remaining $\alpha_{i, j}$ constants (i.e. those with $n+1 \leq i \leq n+v$ and $1 \leq j \leq$ $n+1)$ are randomly chosen, so as to obtain a bijective change of variables.

By rewriting the system $(\mathcal{S})$ with respect to these new variables $y_{i}$, we are led to the following system:

$\left(\mathcal{S}^{\prime}\right)$

$$
\begin{cases}\sum_{i=1}^{n} \beta_{i, 1} y_{i}^{2}+\sum_{i=1}^{n} y_{i} L_{i, 1}\left(y_{n+1}, \ldots, y_{n+v}\right)+Q_{1}\left(y_{n+1}, \ldots, y_{n+v}\right) & =0 \\ \sum_{i=1}^{n} \beta_{i, n} y_{i}^{2}+\sum_{i=1}^{n} y_{i} L_{i, n}\left(y_{n+1}, \ldots, y_{n+v}\right)+Q_{n}\left(y_{n+1}, \ldots, y_{n+v}\right) & =0\end{cases}
$$

where each $L_{i, j}$ is an affine function and each $Q_{i}$ is a quadratic function. 
We then compute $y_{n+1}, \ldots, y_{n+v}$ such that:

$$
\forall i, 1 \leq i \leq n, \forall j, 1 \leq j \leq n+v, L_{i, j}\left(y_{n+1}, \ldots, y_{n+v}\right)=0 .
$$

This is possible because we have to solve a linear system of $n^{2}$ equations and $v$ unknowns, which generally provides at least one solution, as long as $v \geq n^{2}$. We pick one of these solutions. In general, this gives the $y_{i}^{2}$ by Gaussian reduction.

Then, in characteristic 2 , since $x \mapsto x^{2}$ is a bijection, we will then find easily a solution for the $y_{i}$ from this expression of the $y_{i}^{2}$. In characteristic $\neq 2$, it will also succeed when $2^{n}$ is not too large (i.e. when $n \leq 40$ for example). When $n$ is large, there is also a method to find a solution, based on the general theory of quadratic forms. Due to the lack of space, this method will be found in the extended version of this paper.

\section{A Variation with Twice Smaller Signatures}

In the UOV described in section 2, the public key is a set of $n$ quadratic equations $y_{i}=P_{i}\left(x_{1}, \ldots, x_{n+v}\right)$, for $1 \leq i \leq n$, where $y=\left(y_{1}, \ldots, y_{n}\right)$ is the hash value of the message to be signed. If we use a collision-free hash function, the hash value must at least be 128 bits long. Therefore, $q^{n}$ must be at least $2^{128}$, so that the typical length of the signature, if $v=2 n$, is at least $3 \times 128=384$ bits.

As we see now, it is possible to make a small variation in the signature design in order to obtain twice smaller signatures. The idea is to keep the same polynomial $P_{i}$ (with the same associated secret key), but now the public equations that we check are:

$$
\forall i, P_{i}\left(x_{1}, \ldots, x_{n+v}\right)+L_{i}\left(y_{1}, \ldots, y_{n}, x_{1}, \ldots, x_{n+v}\right)=0,
$$

where $L_{i}$ is a linear function in $\left(x_{1}, \ldots, x_{n+v}\right)$ and where the coefficients of $L_{i}$ are generated by a hash function in $\left(y_{1}, \ldots, y_{n}\right)$.

For example $L_{i}\left(y_{1}, \ldots, y_{n}, x_{1}, \ldots, x_{n+v}\right)=\alpha_{1} x_{1}+\alpha_{2} x_{2}+\ldots+\alpha_{n+v} x_{n+v}$, where $\left(\alpha_{1}, \alpha_{2}, \ldots, \alpha_{n+v}\right)=\operatorname{Hash}\left(y_{1}, \ldots, y_{n} \| i\right)$. Now, $n$ can be chosen such that $q^{n} \geq 2^{64}$ (instead $q^{n} \geq 2^{128}$ ). (Note: $q^{n}$ must be $\geq 2^{64}$ in order to avoid exhaustive search on a solution $x$ ). If $v=2 n$ and $q^{n} \simeq 2^{64}$, the length of the signature will be $3 \times 64=192$ bits.

\section{Oil and Vinegar of Degree Three}

\section{The Scheme}

The quadratic Oil and Vinegar schemes described in section 2 can easily be extended to any higher degree. In the case of degree three, the set $(\mathcal{S})$ of hidden equations are of the following type: for all $i \leq n$,

$$
\begin{aligned}
& y_{i}=\sum \gamma_{i j k \ell} a_{j} a_{k}^{\prime} a_{\ell}^{\prime}+\sum \mu_{i j k \ell} a_{j}^{\prime} a_{k}^{\prime} a_{\ell}^{\prime}+\sum \lambda_{i j k} a_{j}^{\prime} a_{k}^{\prime} \\
& +\sum \nu_{i j k} a_{j}^{\prime} a_{k}^{\prime}+\sum \xi_{i j} a_{j}+\sum \xi_{i j}^{\prime} a_{j}^{\prime}+\delta_{i}
\end{aligned}
$$


The coefficients $\gamma_{i j k}, \mu_{i j k \ell}, \lambda_{i j k}, \nu_{i j k}, \xi_{i j}, \xi_{i j}^{\prime}$ and $\delta_{i}$ are the secret coefficients of these $n$ equations. Note that these equations $(\mathcal{S})$ contain no terms in $a_{j} a_{k} a_{\ell}$ or in $a_{j} a_{k}$ : the equations are affine in the $a_{j}$ unknowns when the $a_{k}^{\prime}$ unknowns are fixed.

The computation of the public key, the computation of a signature and the verification of a signature are done as before.

\section{First Cryptanalysis of Oil and Vinegar of Degree Three when $v \leq n$}

We can look at the quadratic part of the public key and attack it exactly as for an Oil and Vinegar of degree two. This is expected to work when $v \leq n$.

Note: If there is no quadratic part (i.e. is the public key is homogeneous of degree three), or if this attack does not work, then it is always possible to apply a random affine change of variables and to try again.

\section{Cryptanalysis of Oil and Vinegar of Degree Three when $v \leq(1+\sqrt{3}) n$ and $K$ Is of Characteristic $\neq 2$ (from an Idea of D. Coppersmith, cf [4])}

The key idea is to detect a "linearity" in some directions. We search the set $V$ of the values $d=\left(d_{1}, \ldots, d_{n+v}\right)$ such that:

$$
\forall x, \forall i, 1 \leq i \leq n, P_{i}(x+d)+P_{i}(x-d)=2 P_{i}(x) \quad \text { (\#). }
$$

By writing that each $x_{k}$ indeterminate has a zero coefficient, we obtain $n \cdot(n+v)$ quadratic equations in the $(n+v)$ unknowns $d_{j}$.

(Each monomial $x_{i} x_{j} x_{k}$ gives $\left(x_{j}+d_{j}\right)\left(x_{k}+d_{k}\right)\left(x_{\ell}+d_{\ell}\right)+\left(x_{j}-d_{j}\right)\left(x_{k}-\right.$ $\left.d_{k}\right)\left(x_{\ell}-d_{\ell}\right)-2 x_{j} x_{k} x_{\ell}$, i.e. $\left.2\left(x_{j} d_{k} d_{\ell}+x_{k} d_{j} d_{\ell}+x_{\ell} d_{j} d_{k}\right).\right)$

Furthermore, the cryptanalyst can specify about $n-1$ of the coordinates $d_{k}$ of $d$, since the vectorial space of the correct $d$ is of dimension $n$. It remains thus to solve $n \cdot(n+v)$ quadratic equations in $(v+1)$ unknowns $d_{j}$. When $v$ is not too large (typically when $\frac{(v+1)^{2}}{2} \leq n(n+v)$, i.e. when $v \leq(1+\sqrt{3}) n$ ), this is expected to be easy. As a result when $v \leq$ approximately $(1+\sqrt{3}) n$ and $|K|$ is odd, this gives a simple way to break the scheme.

Note 1: When $v$ is sensibly greater than $(1+\sqrt{3}) n$ (this is a more unbalanced limit than what we had in the quadratic case), we do not know at the present how to break the scheme.

Note 2: Strangely enough, this cryptanalysis of degre three Oil and Vinegar schemes does not work on degree two Oil and Vinegar schemes. The reason is that - in degree two -writing

$$
\forall x, \forall i, 1 \leq i \leq n, P_{i}(x+d)+P_{i}(x-d)=2 P_{i}(x)
$$

only gives $n$ equations of degree two on the $(n+v) d_{j}$ unknowns (that we do not know how to solve). (Each monomial $x_{j} x_{k}$ gives $\left(x_{j}+d_{j}\right)\left(x_{k}+d_{k}\right)+\left(x_{j}-\right.$ $\left.d_{j}\right)\left(x_{k}-d_{k}\right)-2 x_{j} x_{k}$, i.e. $\left.2 d_{j} d_{k}.\right)$ 
Note 3: In degree two, we have seen that Unbalanced Oil and Vinegar public keys are expected to cover almost all the set of $n$ quadratic equations when $v \simeq \frac{n^{2}}{2}$. In degree three, we have a similar property: the public keys are expected to cover almost all the set of $n$ cubic equations when $v \simeq \frac{n^{3}}{6}$ (the proof is similar).

\section{Another Scheme: HFEV}

In the "most simple" HFE scheme (we use the notations of [14]), we have $b=$ $f(a)$, where:

$$
f(a)=\sum_{i, j} \beta_{i j} a^{q^{\theta_{i j}}+q^{\varphi_{i j}}}+\sum_{i} \alpha_{i} a^{q^{\xi_{i}}}+\mu_{0},
$$

where $\beta_{i j}, \alpha_{i}$ and $\mu_{0}$ are elements of the field $\mathbf{F}_{q^{n}}$. Let $v$ be an integer $(v$ will be the number of extra $x_{i}$ variables, or the number of "vinegar" variables that we will add in the scheme). Let $a^{\prime}=\left(a_{1}^{\prime}, \ldots, a_{v}^{\prime}\right)$ be a $v$-uple of variables of $K$. Let now each $\alpha_{i}$ of (1) be an element of $\mathbf{F}_{q^{n}}$ such that each of the $n$ components of $\alpha_{i}$ in a basis is a secret random linear function of the vinegar variables $a_{1}^{\prime}$, $\ldots, a_{v}^{\prime}$. And in (1), let now $\mu_{0}$ be an element of $\mathbf{F}_{q^{n}}$ such that each one of the $n$ components of $\mu_{0}$ in a basis is a secret random quadratic function of the variables $a_{1}^{\prime}, \ldots, a_{v}^{\prime}$. Then, the $n+v$ variables $a_{1}, \ldots, a_{n}, a_{1}^{\prime}, \ldots, a_{v}^{\prime}$ will be mixed in the secret affine bijection $s$ in order to obtain the variables $x_{1}, \ldots, x_{n+v}$. And, as before, $t\left(b_{1}, \ldots, b_{n}\right)=\left(y_{1}, \ldots, y_{n}\right)$, where $t$ is a secret affine bijection. Then the public key is given as the $n$ equations $y_{i}=P_{i}\left(x_{1}, \ldots, x_{n+v}\right)$. To compute a signature, the vinegar values $a_{1}^{\prime}, \ldots, a_{v}^{\prime}$ will simply be chosen at random. Then, the values $\mu_{0}$ and $\alpha_{i}$ will be computed. Then, the monovariate equations (1) will be solved (in $a$ ) in $\mathbf{F}_{q^{n}}$.

Example: Let $K=\mathbf{F}_{2}$. In HFEV, let for example the hidden polynomial be: $f(a)=a^{17}+\beta_{16} a^{16}+a^{12}+a^{10}+a^{9}+\beta_{8} a^{8}+a^{6}+a^{5}+\beta_{4} a^{4}+a^{3}+\beta_{2} a^{2}+\beta_{1} a+\beta_{0}$, where $a=\left(a_{1}, \ldots, a_{n}\right)\left(a_{1}, \ldots, a_{n}\right.$ are the "oil" variables $), \beta_{1}, \beta_{2}, \beta_{4}, \beta_{8}$ and $\beta_{16}$ are given by $n$ secret linear functions on the $v$ vinegar variables and $\beta_{0}$ is given by $n$ secret quadratic functions on the $v$ vinegar variables. In this example, we compute a signature as follows: the vinegar variables are chosen at random and the resulting equation of degree 17 is solved in $a$.

Note: Unlike UOV, in HFEV we have terms in oil $\times$ oil (such as $a^{17}, a^{12}, a^{10}$, etc), oil $\times$ vinegar (such as $\beta_{16} a^{16}, \beta_{8} a^{8}$, etc) and vinegar $\times \operatorname{vinegar}\left(\right.$ in $\left.\beta_{0}\right)$.

\section{Simulations}

Nicolas Courtois did some simulations on HFEV and, in all his simulations, when the number of vinegar variables is $\geq 3$, there is no affine multiple equations of small degree (which is very nice). See the extended version of this paper for more details. 


\section{Concrete Examples of Parameters for UOV}

At the present, it seems possible to choose for example $n=64, v=128$ (or $v=192$ ) and $K=\mathbf{F}_{2}$. The signature scheme is the one of section 8 , and the length of a signature is only 192 bits (or 256 bits) in this case. More examples of possible parameters are given in the extended version of this paper.

Note: If we choose $K=\mathbf{F}_{2}$ then the public key is often large. So it is often more practical to choose a larger $K$ and a smaller $n$ : then the length of the public key can be reduced a lot. However, even when $K$ and $n$ are fixed, it is always feasible to make some easy transformations on a public key in order to obtain the public key in a canonical way such that this canonical expression is slightly shorter than the original expression. See the extended version of this paper for details.

\section{Concrete Example of Parameters for HFEV}

At the present, it seems possible to choose a small value for $v$ (for example $v=3$ ) and a small value for $d$ (for example $n=77, v=3, d=33$ and $K=\mathbf{F}_{2}$ ). The signature scheme is described in the extended version of this paper (to avoid the birthday paradox). Here the length of a signature is only 80 bits ! More examples of possible parameters are given in the extended version of this paper.

\section{State of the Art (in May 1999) on Public-Key Schemes with Multivariate Polynomials over a Small Finite Field}

Recently, many new ideas have been introduced to design better schemes, such as UOV or HFEV described in this paper. Another idea is to fix some variables to hide some algebraic properties, and another idea is to introduce a few really random quadratic equations and to mix them with the original equations: see the extended version of this paper. However, many new ideas have also been introduced to design better attacks on previous schemes, such as the - not yet published - papers [1], 2], [3], [5]. So the field is fast moving and it can look a bit confusing at first. Moreover, some authors use the word "cryptanalysis" for "breaking" and some authors use this word with the meaning "an analysis about the security" that does not necessary mean "breaking". In this section, we describe what we know at the present about the main schemes.

In the large families of the public key based on multivariate polynomials over a small finite field, we can distinguish between five main families characterized by the way the trapdoor is introduced or by the difficult problem on which the security relies. In the first family are the schemes "with a Hidden Monomial", i.e. the key idea is to compute an exponentiation $x \mapsto x^{d}$ in a finite field for secret key computation. In the second family are the schemes where a polynomial function 
(with more than one monomial) is hidden. In the third family, the security relies on an isomorphism problem. In the fourth family, the security relies on the difficulty of finding the decomposition of two multivariate quadratic polynomials from all or part of their composition. Finally, in the fifth family, the secret key computations are based on Gaussian computations. The main schemes in these families are described in the figure below. What may be the most interesting scheme in each family is in a rectangle.

Family 1: $\quad C^{*}(1985-1995)$

Family 3: IP
Family 2: HFE, (polynomial) Dragons, $H M$


Family 4: (Original) Oil and Vinegar (1997-1998)

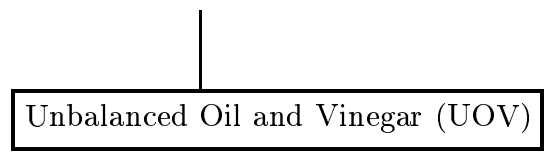

Family 5: 2 Round schemes $(2 R)\left(D^{* *}, 2 R\right.$ with S-boxes, Hybrid $\left.2 \mathrm{R}\right)$

$2 R^{-}$

$-C^{*}$ was the first scheme of all, and it can be seen as the ancestor of all these schemes. It was designed in 12 and broken in 13 .

- Schemes with a Hidden Monomial (such as some Dragon schemes) were studied in [15], where it is shown that most of them are insecure. However, $C^{*--}$ (studied in [20]) is (at the present) the most efficient signature scheme (in time and RAM) in a smartcard. The scheme is not broken (but it may seem too simple or too close to $C^{*}$ to have a large confidence in its security ...).

- HFE was designed in [14. The most recent results about its security are in [1] and [2]. In these papers, very clever attacks are described. However, at the present, it seems that the scheme is not broken since for well chosen and still reasonable parameters the computations required to break it are still too large. For example, the first challenge of US $\$ 500$ given in the extended version of [14] has not been claimed yet (it is a pure HFE with $n=80$ and $d=96$ over $\mathbf{F}_{2}$ ). 
- $\mathrm{HFE}^{-}$is just an HFE where some of the public equations are not published. Due to [1] and [2, it may be recommended to do this (despite the fact that original HFE may be secure without it). In the extended version of [14 a second challenge of US $\$ 500$ is described on a $\mathrm{HFE}^{-}$.

- HFEV is described in this paper. HFEV and $\mathrm{HFEV}^{-}$look very hard to break. Moreover, HFEV is more efficient than the original HFE and it can give public key signatures of only 80 bits !

- $H M$ and $H M^{-}$were designed in [20. Very few analysis have been done in these schemes (but maybe we can recommend to use $H M^{-}$instead of $H M ?)$.

- IP was designed in [14]. IP schemes have the best proofs of security so far (see [19]). IP is very simple and can be seen as a nice generalization of Graph Isomorphism.

- The original Oil and Vinegar was presented in [16] and broken in [10].

- UOV is described in this paper. With IP, they are certainly the most simple schemes.

$-2 R$ was designed in [17] and 18. Due to [3, it is necessary to have at least 128 bits in input, and due to [5], it may be wise to not publish all the (originally) public equations: this gives the $2 R^{-}$algorithms (the efficiency of the decomposition algorithms given in [5] on the $2 R$ schemes is not yet completely clear).

Remark 1: These schemes are of theoretical interest but (at the exception of IP) their security is not directly relied to a clearly defined and considered to be difficult problem. So is it reasonable to implement them in real products ? We think indeed that it is a bit risky to rely all the security of sensitive applications on such schemes. However, at the present, most of the smartcard applications use secret key algorithms (for example Triple-DES) because RSA smartcards are more expensive. So it can be reasonable to put in a low-cost smartcard one of the previous public key schemes in addition to (not instead of) the existing secret key scheme. Then the security can only be increased and the price of the smartcard would still be low (no coprocessor needed). The security would then rely on a master secret key for the secret key algorithm (with the risk of depending on a master secret key) and on a new low-cost public-key scheme (with the risk that the scheme has no proof!!! of security). It can also be noticed that when extremely short signature length (or short block encryption) are required, there is no real choice: at the present only multivariate schemes can have length between 64 and 256 bits.

Remark 2: When a new scheme is found with multivariate polynomials, we do not necessary have to explain how the trapdoor has been introduced. Then we will obtain a kind of "Secret-Public Key scheme" ! The scheme is clearly a Public Key scheme since anybody can verify a signature from the public key (or can encrypt from the public key) and the scheme is secret since the way to compute the secret key computations (i.e. the way the trapdoor has been introduced) has not been revealed and cannot be guessed from the public key. For example, we could have done this for HFEV (instead of publishing it). 


\section{Conclusion}

In this paper, we have presented two new public key schemes with "vinegar variables": UOV and HFEV. The study of such schemes has led us to analyze very general properties about the solutions of systems of general quadratic forms. Moreover, from the general view presented in section 13, we see that these two schemes are at the present among the most interesting schemes in two of the five main families of schemes based on multivariate polynomials over a small finite field. Will this still be true in a few years?

\section{References}

1. Anonymous, Cryptanalysis of the HFE Public Key Cryptosystem, not yet published.

2. Anonymous, Practical cryptanalysis of Hidden Field Equations (HFE), not yet published.

3. Anonymous, Cryptanalysis of Patarin's 2-Round Public Key System with S Boxes, not yet published.

4. D. Coppersmith, personal communication, e-mail.

5. Z. Dai, D. Ye, K.-Y. Lam, Factoring-attacks on Asymmetric Cryptography Based on Mapping-compositions, not yet published.

6. J.-C. Faugere, personal communication.

7. H. Fell, W. Diffie, Analysis of a public key approach based on polynomial substitutions, Proceedings of CRYPTO'85, Springer-Verlag, vol. 218, pp. 340-349

8. M. Garey, D. Johnson, Computers and Intractability, a Guide to the Theory of NP-Completeness, Freeman, p. 251.

9. H. Imai, T. Matsumoto, Algebraic Methods for Constructing Asymmetric Cryptosystems, Algebraic Algorithms and Error Correcting Codes (AAECC-3), Grenoble, 1985, Springer-Verlag, LNCS n ${ }^{\circ} 229$.

10. A. Kipnis, A. Shamir, Cryptanalysis of the Oil and Vinegar Signature Scheme, Proceedings of CRYPTO'98, Springer, LNCS n ${ }^{\circ} 1462$, pp. 257-266.

11. R. Lidl, H. Niederreiter, Finite Fields, Encyclopedia of Mathematics and its applications, volume 20, Cambridge University Press.

12. T. Matsumoto, H. Imai, Public Quadratic Polynomial-tuples for efficient signatureverification and message-encryption, Proceedings of EUROCRYPT'88, SpringerVerlag, pp. 419-453.

13. Jacques Patarin, Cryptanalysis of the Matsumoto and Imai public Key Scheme of Eurocrypt'88, Proceedings of CRYPTO'95, Springer-Verlag, pp. 248-261.

14. J. Patarin, Hidden Fields Equations (HFE) and Isomorphisms of Polynomials (IP) : Two New Families of Asymmetric Algorithms, Proceedings of EUROCRYPT'96, Springer, pp. 33-48.

15. Jacques Patarin, Asymmetric Cryptography with a Hidden Monomial, Proceedings of CRYPTO'96, Springer, pp. 45-60.

16. J. Patarin, The Oil and Vinegar Signature Scheme, presented at the Dagstuhl Workshop on Cryptography, september 1997 (transparencies).

17. J. Patarin, L. Goubin, Trapdoor One-way Permutations and Multivariate Polynomials, Proceedings of ICICS'97, Springer, LNCS nº1334, pp. 356-368.

18. J. Patarin, L. Goubin, Asymmetric Cryptography with S-Boxes, Proceedings of ICICS'97, Springer, LNCS n ${ }^{\circ} 1334$, pp. 369-380. 
19. J. Patarin, L. Goubin, N. Courtois, Improved Algorithms for Isomorphisms of Polynomials, Proceedings of EUROCRYPT'98, Springer, pp. 184-200.

20. J. Patarin, L. Goubin, N. Courtois, $C_{-+}^{*}$ and HM: Variations Around Two Schemes of T. Matsumoto and H. Imai, Proceedings of ASIACRYPT'98, Springer, pp. 35-49.

21. A. Shamir, A simple scheme for encryption and its cryptanalysis found by $D$. Coppersmith and J. Stern, presented at the Luminy workshop on cryptography, september 1995. 